Vernay, D.G., Raphael, B. \& Smith, I.F.C. "A model-based data-interpretation framework for improving wind predictions around buildings" Journal of Wind Engineering and Industrial Aerodynamics, 145, 2015 pp.219-228 http://dx.doi.org/10.1016/j.jweia.2015.06.016

\title{
A MODEL-BASED DATA-INTERPRETATION FRAMEWORK FOR IMPROVING WIND PREDICTIONS
} AROUND BUILDINGS

Didier G. Vernay ${ }^{\text {a,c,*, Benny Raphael }}{ }^{\text {b }}$ and Ian F.C. Smith ${ }^{\text {a,c }}$

a) Future Cities Laboratory, ETH Zurich, Zurich, Switzerland.

b) Civil Engineering Department, Indian Institute of Technology, Madras, India.

c) Applied Computing and Mechanics Laboratory, School of Architecture, Civil and Environ $\$$ tal Engineering (ENAC), EPFL, Lausanne, Switzerland.

* Cortespon

* Corresponding author: Didier G. Vernay, Future Cities Laboratory, Singapore-ETH Centre 1 CREATE Way \#06-01

CREATE Tower Singapore 138602. Tel.: +65 9723 1127, E-mail: didier.vernay@epfl.ch

Abstract:

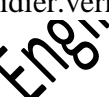

Although Computational Fluid Dynamics (GE) simulations are often used to assess wind conditions around buildings, the accuracy of suck mulations is often unknown. This paper proposes a datainterpretation framework that unultiple simulations in combination with measurement data to improve the accuracy of wind Predictions. Multiple simulations are generated through varying sets of parameter values. Sets parameter values are falsified and thus not used for predictions if differences between measuredent data and simulation predictions, for any measurement location, are larger than an estimare unceratities at sensor locations. The framework accounts for time-dependent and spatially-distributed C

fodelling uncertainties that are present in CFD simulations of wind. The framework is applied to the case study of the CREATE Tower located at the National University of Singapore. Values for timedependent inlet conditions, as well as values for the roughness of surrounding buildings, are identified with measurements carried out around the CREATE Tower. Results show that, on average, ranges of 
horizontal wind-speed predictions at an unmeasured location have been decreased by $65 \%$ when measurement data are used.

Keywords: Computational Fluid Dynamics (CFD); wind modelling; field measurements; data interpretation; multi-model reasoning

\section{1) Introduction}

Wind around buildings affects the comfort and health of residents as well as the energy ofmption of buildings, particularly in tropical climates. For example, the convective heat flo building façade, influencing energy consumption of buildings, depends on the surroundind [1]. Wind can also be harnessed for natural ventilation of buildings [2]. Computatian fluid Dynamics (CFD) simulations have been widely used to simulate wind around and trough buildings [3, 4]. Although guidelines have been established to improve simulation predgens [5], large discrepancies remain when simulation predictions are compared to field simulation predictions are usually not quantified

The steady Reynolds-averaged Navier-Stat (RANS) equations are usually employed in CFD simulations to describe the fluid-fto thehavior. These equations are time-averaged or ensembleaveraged equations of the flui $20 \mathrm{w}$ motion. Large discrepancies have been observed in wakes of buildings when predictiors by RANS-based simulations are compared with wind-tunnel experiments

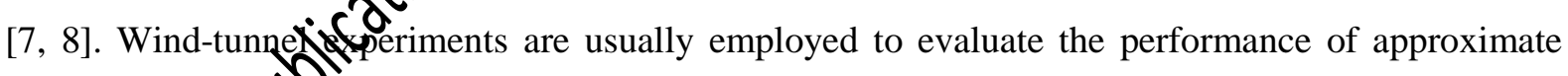
equations of fufferlow solved in CFD simulations because values of parameters are known (e.g. inlet conditiog and surface roughness). Large Eddy Simulation (LES) is an alternative strategy for mgchling fluid-flow behavior in which time-dependent predictions are computed. LES has been found to provide better agreement with wind-tunnel experiments than RANS-based simulations [7].

Thermal processes may affect the wind behavior around buildings, especially in street canyons which can be subject to combinations of low wind speeds and high differential heating between surfaces [9]. However, if thermal processes are implemented into the CFD model, modeling complexity is increased $[3,10]$ along with the number of parameters that cannot be easily estimated, such as the 
thermal properties of surfaces. Therefore, thermal processes are not often included in CFD simulations. Effects of thermal processes have been evaluated by using field measurements [9]; by simulating thermal processes with CFD simulations [11]; or by using wind-tunnel experiments with a heated floor [12]. However, the effects have been estimated for standard building configurations (street canyons) and they are likely to vary for other topologies.

Model-based data interpretation has the potential to improve the accuracy of simulation predictons through the use of a population of CFD simulations and measurement data. In model bsted datainterpretation approaches, many model instances (simulation instances) are getated through assigning sets of parameter values to a model class. In this work, the modedass is a CFD model with un-assigned parameter values. Measurement data are used to estimats of parameter values by solving an inverse problem. The inverse problem involves esting sets of parameter values by comparing measurement data with predictions of model instes. Several approaches are described in following chapters.

Model calibration, in which an "optimal" mode found by minimizing the sum of the squared difference between simulation prediction ond measurement data, is not appropriate because there rarely is a single answer to the invers froblem. Many set(s) of parameter values within a model class might give same responses sensor locations in complex systems [13]. Such ambiguities are amplified by measuremand modelling uncertainties. Modeling uncertainties refer to uncertainties (probability distrins of errors) in the model class (e.g uncertainties associated with RANS equations) values of parameters, which compensate modeline measurement errors at sensor locations. Therefore, the "optimal” model is conditional orefonsor locations (and modeling errors at those locations). Furthermore, calibration approaches do not provide information that can lead to estimates of uncertainties of subsequent predictions [14].

Bayesian inference identifies conditional probability distributions of parameter values given measurement data [15]. Probability distributions are required to represent measurement and modeling uncertainties at sensor locations. Uncertainties in CFD simulations are difficult, if not impossible to 
determine precisely. If incorrect probability distributions are defined, it may lead to over-conditioning of parameter values [14]. Furthermore, modeling errors are often systematic and this introduces additional error correlations between measurement locations [16, 17]. Implementation of Bayesian inference requires a complete knowledge of all correlations in order to avoid biased predictions. In wind modeling, the values of such correlations are unknown.

An alternative is to use a model-falsification approach, such as error-domain model falsificatigent 18] and Generalized Likelihood Uncertainty Estimation (GLUE) [14], in which incords sets of parameter values are falsified using measurement data. Only bounds of measurentand modeling uncertainties are needed. Error-domain model falsification has been developen the application of bridge diagnosis and leak detection in water networks. Error-domain foldel falsification involves falsification of model instances for which differences between predictions, for any measurement location, are larger than estimate of uncertainty bounds; the bounds are defined by combining measurement unchlinies and modeling uncertainties at that location. When the entire set of model instances lsified, the model class is incorrect. This could

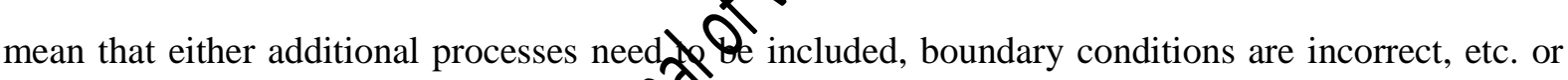
modeling and measurement uncertaing have been underestimated. In this way, model falsification provides a way to test the validit model classes.

The main objective of appropriate for thistification of parameter values of CFD simulations, and subsequent predictions at unmeasure fosations. The framework is based on error-domain model falsification. Improvements have bedhade to error-domain model falsification in order to reproduce time variability (at the scale (nifinutes) of wind through allowing identification of different sets of inlet conditions at different times. In this framework, time-dependent inlet conditions as well as the roughness of the surrounding buildings are identified using time series of measurement data.

Modelling and measurement uncertainties affect the information content of measurement data. A systematic methodology to evaluate modeling uncertainties is proposed that recognizes their time- 
dependent and spatially-distributed characteristics. The final objective is to apply the methodology to the case study of the "CREATE Tower". The CREATE Tower is a 16-storey building located at the National University of Singapore.

The structure of the paper is as follows. In the next section, the model-based data-interpretation framework is described. Section 3 introduces the case study and the model class including the parameters requiring identification. The experimental setup is presented in Section 4. Seg 5 presents a methodology to estimate modeling uncertainties that can be incorporated to modelbased data-interpretation framework. The model-based data-interpretation framgure is applied in Section 6 using simulation predictions, measurement data and knowledge modeling uncertainties. The paper ends with a discussion of the results alans for future work.

2) Methodology

This section presents the model-based data-interpreto framework used to identify sets of parameter values of the CFD simulation and priables at unmeasured locations. This framework is based on error-domain modsification which has been found to be useful in applications of bridge diagnosis and detection in water networks [16, 18]. In such systems, parameter values are identifiesing measurements carried out only at specific times. In the assessment of wind behavieNaround buildings, parameter values of CFD simulations need to be identified dynamically

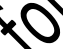

21) rror-domain model falsification

Errog monain model falsification involves generating sets of model instances $M\left(\boldsymbol{\theta}_{j}\right)$ through assigning a combination of parameter values $\boldsymbol{\theta}_{j}=\left[\theta_{1}, \ldots, \theta_{p}\right]_{j}$ to a model class $M$ with $j \in\left\{1, \ldots, n_{m}\right\} . p$ is the number of parameters requiring identification and $n_{m}$ is the number of model instances. When correct sets of parameter values $\boldsymbol{\theta}^{*}$ are assigned to the model class, the predicted value of an output variable of the model instance $M\left(\boldsymbol{\theta}^{*}\right)$ differs from the real value (Q) 
by the modelling error $\grave{O}_{\text {model }}$. Modelling errors are errors associated with the model class. The real value is also equal to the measured value $y$ plus a measurement error $\grave{O}_{\text {measure }}$. This is expressed in Equation (1).

$M\left(\boldsymbol{\theta}^{*}\right)+\grave{O}_{\text {model }}=Q=y+\grave{O}_{\text {measure }}$

Equation (2) is derived by rearranging the terms in Equation (1). The difference between the and the measured value is equal to the difference between the measurement and the modefor error.

$M\left(\boldsymbol{\theta}^{*}\right)-y=\grave{o}_{\text {measure }}-\grave{o}_{\text {model }}$

However, errors are seldom known in environmental systems. Errors represented with probability distributions (uncertainties), which are often assumed uniform [19]. Measurement and modelling uncertainties are combined usique [20]. Threshold bounds $\left[T_{\text {low }}, T_{\text {high }}\right]$ are defined using the combipertainty and a confidence level of $\phi=95 \%$ as presented in Figure 1.

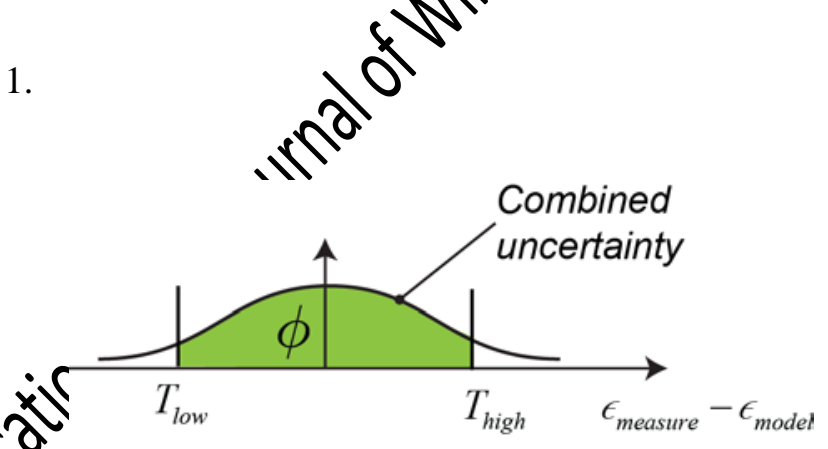

Figure 1: Thres o $)$ bounds determined with the combined uncertainty and a confidence level

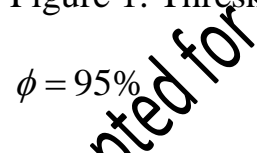

$\times$

cel

model instance $M\left(\boldsymbol{\theta}_{j}\right)$ is candidate if Equation (3) is satisfied. Otherwise, the model instance is

falsified $\left(\boldsymbol{\theta}_{j} \neq \boldsymbol{\theta}^{*}\right)$.

$T_{\text {low }} \leq M\left(\boldsymbol{\theta}_{j}\right)-y \leq T_{\text {high }}$ 
Compared with typical Bayesian approaches (zero-mean Gaussian probability forms), this approach is more robust when error correlations are not known [17].

\section{2) Application to wind modeling}

In this work, the parameters requiring identification are the inlet wind speed $U_{\text {inlet, } 16 \mathrm{~m}}$ at $16 \mathrm{~m}$ height, the inlet wind direction $\vartheta_{\text {inlet }}$ and the sand-grain roughness of the surrounding buildings $\left.\boldsymbol{\theta}=\left[U_{\text {inlet }, 16 m}, \vartheta_{\text {inlet }}, k_{s}\right]\right)$.The output variables of interest are the horizontal wind speeds 1 dind wind directions $\vartheta$ at sensor location $i \in\left\{1, \ldots, n_{s}\right\}$ and at unmeasured locations of ind where $n_{s}$ is the number of sensor locations.

Modeling uncertainties are time dependent and spatially distribsection 5). Thus, modelling uncertainties (and threshold bounds $\left[T_{l o w, i, j, k, t}, T_{h i g h, i, j, k, t}\right]$ ) location $i$, for each model instance $j$, for each compared variable $j\{(U, \vartheta\}$ and at each time step $t$. At time step $t$ , model instances are candidate models if, fordach and every sensor location and for each and every compared variable, the difference betwe measured and the predicted value falls inside the interval $\left[T_{l o w, i, j, k, t}, T_{h i g h, i, j, k, t}\right]$.

$$
\forall i \in\left\{1, \ldots, n_{s}\right\} \text { and } \forall j_{i}
$$

Where $M($ is the predicted value of the variable $k$ at sensor location $i$ by the model instance $j$ and value of the variable $k$ at sensor location $i$ at time step $t$.

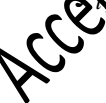

Model instances are steady-state models. At each time step, specific set(s) of parameter values are identified in order to represent the dynamic behavior of wind.

2.3)Wind predictions at unmeasured locations 
Each candidate model is assumed to be equally probable. A discrete distribution of values can be predicted with the set of candidate models for each variable $k$, at each unmeasured location of interest $l$ and at each time step $t$. These distributions correspond to uncertainties associated with parameter values, which have been reduced through falsification of incorrect sets of parameter values.

In order to obtain unbiased predictions, modelling uncertainties need to be combined to these distributions as expressed in Equation (5). For each candidate-model prediction, 2000 samp are drawn from distributions of modelling-uncertainty sources in order to build predictive digosttions at unmeasured locations.

$P_{l, k, t}=M\left(\theta_{j^{*}}\right)_{l, k}+\grave{O}_{\operatorname{model} l, j^{*}, l, k, t}$

Where $P_{l, k, t}$ is the predictive distribution of the variable $k$ at the location $l$ and at the time step t. $M\left(\theta_{j^{*}}\right)_{l, k}$ is the prediction of the variable candidate model $j^{*}$ at the unmeasured location $l . \grave{O}_{\text {model } l, j^{*}, l, k, t}$ is a rando $v$ atiable representing the modelling uncertainty of the candidate model $j^{*}$ in the prediction of variable $k$ at the unmeasured location $l$ and at the time step $t$.

Ranges of predictions corsponding to any confidence level (for example 50, 70 and 95\%) can be computed using thetictive distributions.

3) Nufinecal simulations

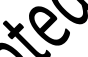

CFes nulations have been executed using the commercial software FLUENT 14.5 [21] in order to are

smulate possible wind behavior around the CREATE Tower. The CREATE Tower is a 16-storey building located at the National University of Singapore.

The 3D models of the buildings have been built using photogrammetry techniques [22]. The resolution of the 3D model of the CREATE Tower is approximatively $5 \mathrm{~cm}$. A lower resolution is employed for the surrounding buildings. The surrounding buildings of the CREATE Tower have been 
modelled following best practice guidelines [23]. If the distance from a building of height $H$ to the CREATE Tower is lower than $6 H$, the building is explicitly modelled. The size of the computational domain has also been determined using best practice guidelines [23], creating a computational domain with dimensions $L \times W \times H=2233 m \times 1144 m \times 368 m$.

CutCell Meshing has been employed to generate the grid. CutCell Meshing generates a high percentage of hexahedral cells which provide better iterative convergence than tetrahedral ceht.

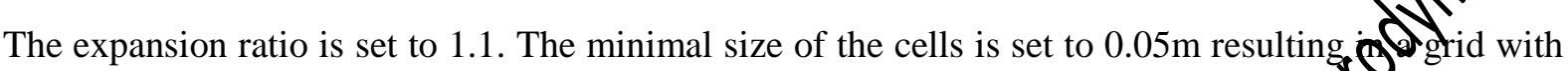
$13.1 \times 10^{6}$ cells. Results have been compared with a finer grid in which the minits of the cells has been reduced to $0.03 \mathrm{~m}$. In average over 187 locations close to the sensor locations mentioned in Section 4), the mean difference byeen horizontal wind speeds predicted with the two grids corresponds to $1.1 \%$ of the inlet wine ${ }_{d}$ at the same height. The finer grid does not improve significantly the results and, thereforgat not been selected.

The 3D steady RANS equations are used in the nass to describe the flow behavior around the CREATE Tower. The Realizable $k-\varepsilon$ me employed to provide closure with two additional transport equations [24]. The first new variable is the turbulence kinetic energy $k$ and the second is the turbulence dissipare $\varepsilon$. Isothermal conditions were imposed. More sophisticated model class can be emplos to simulate the effect of thermal processes on the wind behavior. However, it would the modelling complexity and would require the definition of new parameter valueg dech as the thermal properties of the surfaces as well as the sun radiation which vary with resper to time. Thus, isothermal conditions have been considered in the model class. Nevefless, the effect of thermal processes on wind predictions is evaluated using statistical C Thods on measurement data taken at different times of the day (and night) in Section 5.3 in order to identify reliable sets of parameter values and predict reliable wind variables at unmeasured locations.

The Coupled algorithm is employed for pressure-velocity coupling. A second-order discretization scheme is used to interpolate pressure from values at cell centers. The convergence criteria, based on 
the scaled residuals, are set to $10^{-4}$ for all variables. Before simulations were terminated, the predicted values of the variables of interest were constant. Thus, the solution was regarded as converged [25].

A user-defined function (UDF) in FLUENT is employed to define a vertical profile of wind speed $U_{\text {inlet }}$, turbulence kinetic energy $k_{\text {inlet }}$ and turbulence dissipation rate $\varepsilon_{\text {inlet }}$ at the inlet of the computational domain. For the $k-\varepsilon$ model, profiles have been proposed by Richards and Hof [26]. They are expressed in Equation(6), Equation (7) and Equation (8).

$U_{\text {inlet }}(y)=\frac{\mathrm{u}_{\mathrm{ABL}}^{*}}{\kappa} \ln \left(\frac{y+y_{0}}{y_{0}}\right)$

$k_{\text {inlet }}(y)=\frac{\mathrm{u}_{\mathrm{ABL}}^{*}{ }^{2}}{\sqrt{\mathrm{C}_{\mu}}}$

$\varepsilon_{\text {inlet }}(y)=\frac{\mathrm{u}_{\mathrm{ABL}}^{*}{ }^{3}}{\kappa\left(y+y_{0}\right)}$

where $y$ is the height coordinate, $y_{0}$ is constant, $\mathrm{u}_{\mathrm{ABL}}^{*}$ is the atmospheric bofdary layer (ABL) friction velocity and $\mathrm{C}_{\mu}$ is a model constant of the $k-\varepsilon$ model.

The flow behaviox walls is modeled with the standard-wall function [27]. The sand-grain roughness $k_{\mathrm{s}}$ nfeds to be defined at wall surfaces in FLUENT. A relationship between the sand-grain roughise $k_{s}$ and the roughness length $y_{0}$ (commonly used in wind engineering) has been Q

Sylished for the standard-wall function in FLUENT by Blocken et al. [28]. This relationship is expressed in Equation (9).

$k_{s}=\frac{9.793 y_{0}}{C_{s}}$ 
Where $C_{s}$ is the roughness constant. The value of $k_{s}$ cannot be larger than $y_{p}$, which is the distance between the wall and the centroid of the wall-adjacent cell. Therefore, $C_{s}$ may need to be adjusted in FLUENT to satisfy Equation (9).

The terrain of the computational domain is decomposed into two surfaces. The first surface is the terrain where the surrounding buildings are explicitly modelled. The second surface is the upstrin? terrain where the buildings are implicitly modelled using an equivalent roughness lengt ${ }_{0}$. The roughness length used to model the upstream terrain is set to $y_{0}=0.8 \mathrm{~m}$, which repents regularlybuilt large town [29]. By using Equation (9), the sand-grain roughness and roughness constant have been set to $k_{s}=1.33$ and $C_{s}=6$. The roughness length imposedons terrain is also used for the definition of inlet profiles (Eqs. (6)-(8)) in order to aivid inintended streamwise gradient associated with roughness modification in the upstream pard the computational domain [28]. The terrain where the surrounding buildings are explig modelled is set to $y_{0}=0.24 \mathrm{~m}$. Symmetry boundary conditions are imposed on the sidgand on the top of the computational domain. Zeropressure boundary condition is imposed outlet.

The parameters requiring idention are the inlet wind speed at $16 \mathrm{~m}$ height $U_{\text {inlet, } 16 \mathrm{~m}}$, the inlet wind direction $\vartheta_{\text {inlet }}$ and the $M\left(\boldsymbol{\theta}_{j}\right)$ has beeperted through assigning sets of parameter values $\boldsymbol{\theta}_{j}=\left[U_{\text {inlet }, 16 m}, \vartheta_{\text {inlet }}, k_{s}\right]_{j}$ to the moded $M$ described in previous paragraphs. These parameters have been selected because theye the highest impact on wind predictions and are difficult to estimate.

Wind speeds and wind directions at the inlet of the CFD simulation are sensitive parameters and are difficult to estimate in urban areas [30]. Buildings and streets in the area of interest are modelled with a certain degree of geometrical simplification in CFD simulations. A roughness is imposed on these surfaces to implicitly model those simplifications. This roughness is difficult to estimate and may have a strong influence on predictions of wind speeds (variations up to $25 \%$ for mean wind speeds) 
[31]. In the proposed framework, representative inlet conditions and roughness of the surrounding buildings are identified using measurement data from sensors located around the CREATE Tower.

Grid-based sampling is used to select sets of parameter values uniformly within the parameter space for the generation of model instances. Table 1 presents the maximal and minimal values of parameters as well as their discretization intervals. CFD simulations have been executed for each combination of inlet wind direction $\vartheta_{\text {inlet }}$ and roughness of the surrounding buildings $k_{s}$. A single value of inks speed $U_{\text {inlet,16m }}$ is used in the CFD simulations. A total of 48 simulations have been exaged in batch mode using 12 processes in parallel on a Windows Server 2012 containing four E54607 2.20GHz processors and 64 GB memory which requires apprdhatively 192 hours of execution time. When a new value of inlet wind direction is assigneg the model class, the outside box of the computational domain modifies its orientation, leadin the generation of a new grid.

Wind predictions for other inlet wind speeds are obta observed that same amplification factor of wind and same wind directions are predicted at one location when the value of inlet wind spens varied. In total, wind predictions of 768 model instances are obtained although only 48 simulations have been executed.

Horizontal wind speeds and windirections are predicted at sensor locations for each model instance. Figure 2 presents the horital wind speeds and wind directions at 30 meter height predicted by one model instance.

Table 1: Matulat and maximal values of parameters requiring identification as well as discretization

\begin{tabular}{|c|c|c|c|}
\hline Wind direction at the inlet $\vartheta_{\text {inlet }}$ form North [ $\left.{ }^{\circ}\right]$ & 0 & 337.5 & 22.5 \\
\hline Wind speed at the inlet $U_{\text {inlet, } 16 m}$ (at $16 \mathrm{~m}$ height) [m/s] & 0.5 & 8 & 0.5 \\
\hline
\end{tabular}




\begin{tabular}{|l|l|l|l|}
\hline Sand-grain roughness of surrounding buildings $k_{s}[\mathrm{~m}]$ & 0.02 & 0.32 & 0.15 \\
\hline
\end{tabular}

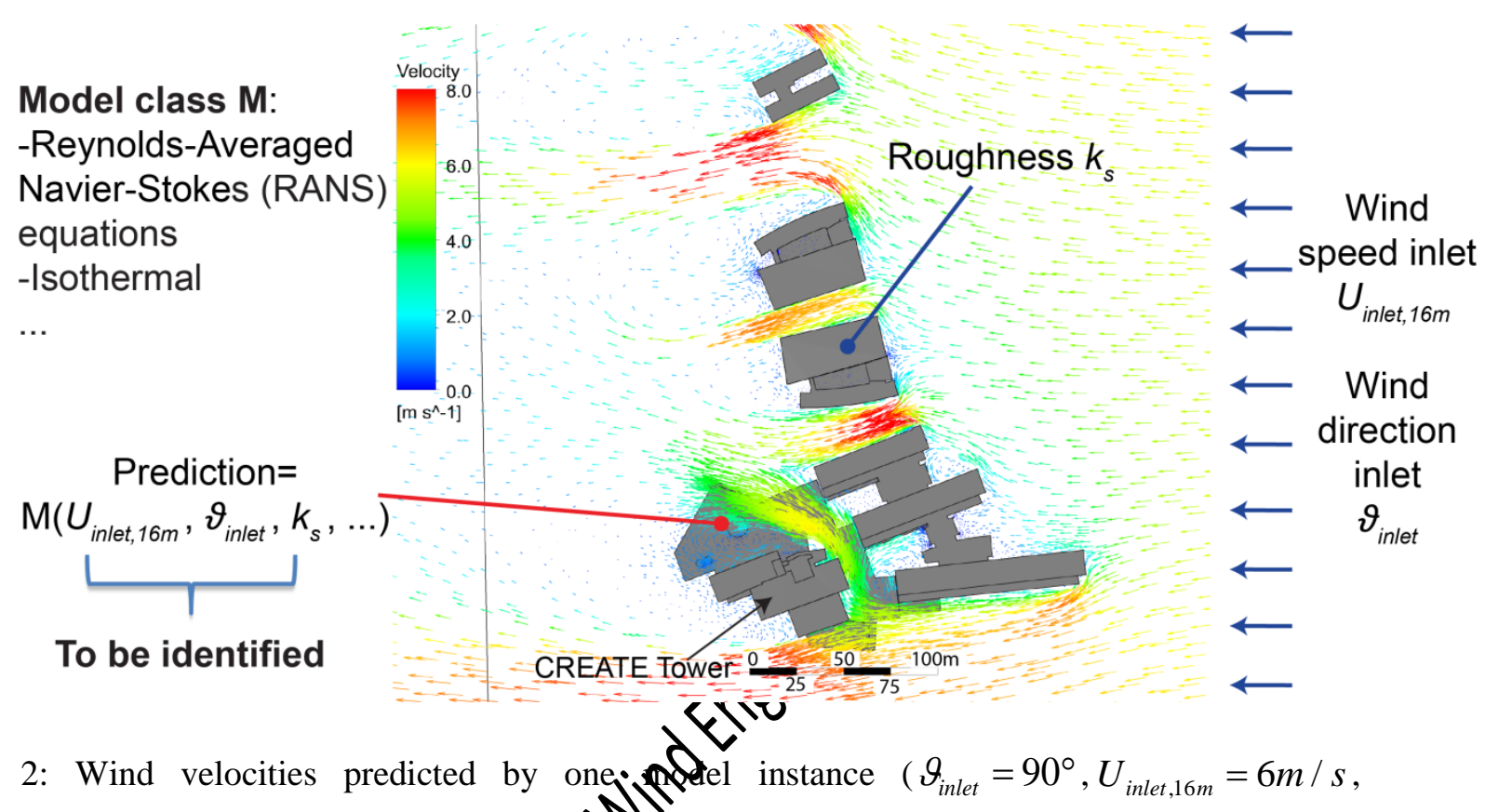

Figure 2: Wind velocities predicted by one pelel instance $\left(\vartheta_{\text {inlet }}=90^{\circ}, U_{\text {inlet }, 16 \mathrm{~m}}=6 \mathrm{~m} / \mathrm{s}\right.$, $k_{s}=0.17 \mathrm{~m}$ ) at 30 meter height determined tough a CFD simulation (top view) and description of the system.

4) Experimental setup and

Measurements were cant four from February 14. 2014 to March 21. 2014 during the North-East monsoon season.

A sensor ment methodology was applied in order to define measurement locations that should bring largest amount of information on the wind behavior around the CREATE Tower [32]. ce

PSsible locations were limited to the balconies and terraces of the CREATE Tower. From the 187

possible locations defined initially, the 8 best locations have been determined. Figure 3 presents the possible locations of weather stations as well as their actual locations. Seven weather stations are used to falsify incorrect sets of model instances (sensor S1 to S7) and the last weather station is used to test 
the framework (sensor S8). The weather stations were deployed around the CREATE Tower at different floor levels (L3, L7 and L13).

Four Davis weather stations, each equipped with a wind-cup anemometer and a wind vane were used to measure horizontal wind speeds and wind directions (sensor S1 to S4) and four HOBO weather stations equipped with a wind-cup anemometer, a wind vane and a temperature sensor were used to measure horizontal wind speeds, wind directions and temperatures (sensor S5 to S8).

A data logger was installed on each weather station to store measurement data. Averoe values of measurement data were logged every minute. The resolutions of the Davis weatherations are $0.1 \mathrm{~m} / \mathrm{s}$ for horizontal wind-speed measurements and $22.5^{\circ}$ for wind-direction measurements. The starting threshold is $0.4 \mathrm{~m} / \mathrm{s}$. The resolutions of the HOBO weather stations $200.19 \mathrm{~m} / \mathrm{s}$ for horizontal windspeed measurements and $1.4^{\circ}$ for wind-direction measurements. The starting threshold is $0.5 \mathrm{~m} / \mathrm{s}$.

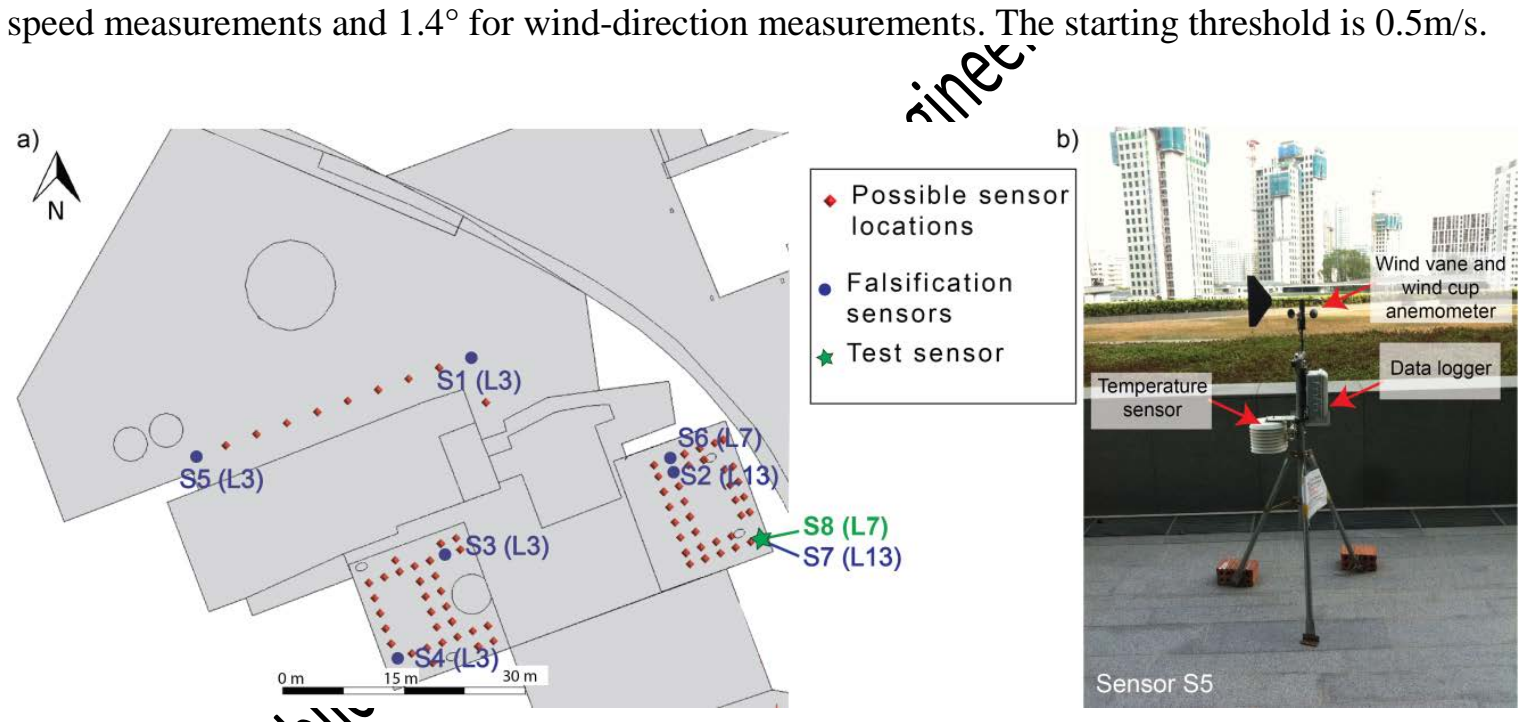

Figure 3: a) Mearthement locations around the CREATE Tower (top view) and b) description of the

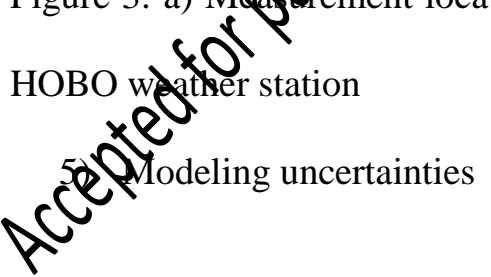

Modelling uncertainties at sensor locations affect the information content of measurement data. This section proposes strategies to estimate main sources of uncertainties and to combine them in order to define threshold bounds used for falsification of incorrect model instances. 
5.1) Uncertainties associated with RANS-based simulation in the predictions of mean wind variables

LES has been found to be more accurate than RANS-based simulation in regions of flow separation and recirculation when compared to wind-tunnel experiments because flow is highly unsteady in these regions [7]. However, LES takes two order of magnitude longer to execute than a RANS-based simulation [33] and, therefore, it has not been employed for the generation of model ingares (Section 3). Uncertainties associated with RANS-based simulation are estimated by dodmparing predictions of a RANS-based simulation with those of a LES. Horizontal wind and wind directions predicted at 10000 locations around the CREATE Tower with the compared with mean values of LES predictions.

The geometry and grid settings described in Section 3 are useditor both LES and the RANS-based simulation. The same wind conditions are defined at thes of both simulations. The turbulence kinetic energy imposed at the inlet of the RANS-basedimulation is reproduced in LES by imposing a time-dependent wind-speed profile using the method [34] in FLUENT 14.5. 190 vortices are used in the vortex method because it herovided good results in previous studies on the wind behavior around a wall-mounted cubs 935 ]. In LES, the dynamic Smagorinsky model is employed to model the small eddies of the [36].

A zero sand-grain roygess is imposed on all surfaces of the RANS-based simulation in order to be consistent with In order to avoid unintended streamwise gradient in the upstream part of the computat domain, a small roughness length $\left(y_{0}=0.001 \mathrm{~m}\right)$ is used to define the inlet profiles (Egefor)-(8)), which is different from the roughness length used to model the upstream terrain in the generation of model instances (Section 3). In this section, the goal is to estimate the uncertainties associated with steady RANS equations by comparing RANS predictions with equivalent LES predictions. The goal is not to predict the wind velocity accurately for the selected case study. Since the uncertainties in the RANS predictions are defined as a function of the inlet wind speed, avoiding 
unintended streamwise gradients in the upstream part of the computational domain leads to consistent estimations of uncertainties.

Responses of the RANS-based simulation are employed as initial conditions for LES. In LES, the time step size is set to $0.25 \mathrm{~s}$. After $1 \mathrm{~h}$ of real time, mean values of LES do not vary significantly.

Figure 4 presents differences between mean responses of LES and responses of the RANS-bared simulation with respect to the amplification factor of wind speeds predicted with the RAmsosased simulation. The amplification factor of wind speeds is defined as the ratio between De $_{2}$ predicted horizontal wind speed $U$ and the inlet wind speed at the same height $U_{0}$. It is thy streamwise gradients in the upstream part of the computational domain.

Differences in the predictions of horizontal wind speeds are highen regions of low amplification factors of wind speeds (Figure 4a). Differences in the prefetions of horizontal wind speeds are defined as a percentage of the inlet wind speed at ths stre height $U_{0}$. Indeed, in a previous paper, LES and RANS-based predictions around a sigubical building have been compared for two inlet wind speeds [37]. Differences in the predi ns of horizontal wind speeds between LES and RANSbased simulations have been found tofe proportional to $U_{0}$ [37].

Large variations in the predictions of wind directions are observed in regions of low amplification factors of wind spegrothis is in agreement with results found using a single cubical building [37]. This originates of $d$ the over-estimation of the region of reverse flow in wakes of buildings with RANS-bad simulations. Therefore, RANS-based simulations may predict reverse flow at locations whection do not. Model calibration mentioned in Section 1 is especially hazardous when sensors are Cated in regions of high uncertainties because the calibrated set of parameter values may compensate with possible high errors in the model class.

Two regions are defined in the model-based data-interpretation framework in order to acknowledge for the spatial variability of modeling uncertainties; the first region is defined by amplification factors of wind speeds that are lower than 0.33 and the second region is defined by amplification factors of 
wind speeds that are higher than or equal to 0.33 . Locations of those regions vary for each model instance. Based on the distributions of differences, this source of uncertainty is described with a normal distribution for each flow variable and for each region.

a)

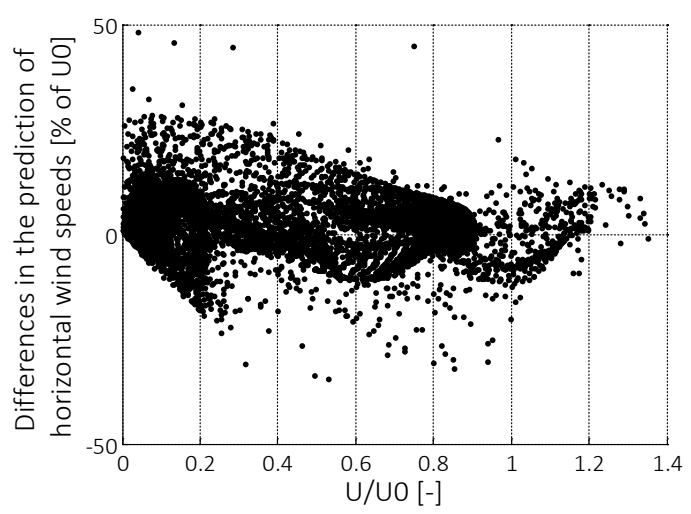

b)

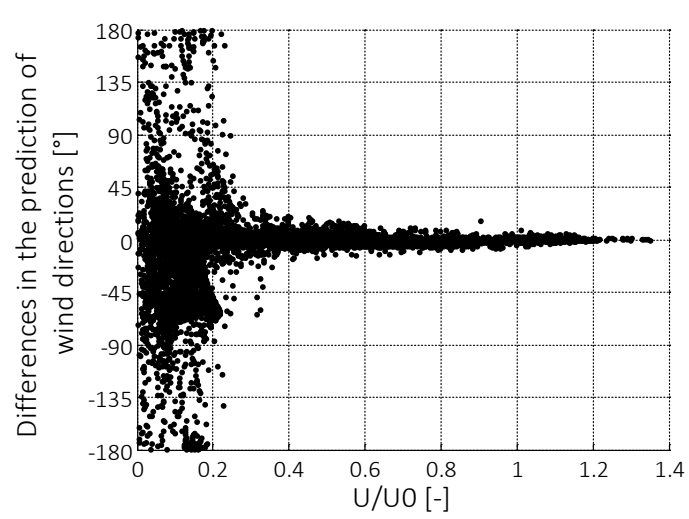

Figure 4: Differences between LES and a RANS-based simula 4 in the predictions of a) horizontal wind speeds and b) wind directions with respect to the speeds

\section{2) Uncertainties associated with}

In the model-based data-interpretation to average measurement data. Defineng the averaging window is a challenging task because of the presence of two conflicting octives: 1) the averaging window should be short enough to be able to assume constant wind of the RANS-based simulations used in the model-based data-interpretatigntewewn 2) the averaging window should also be long enough in order to avoid fluctuation $\&$ wind variables associated with turbulence at locations of interest. Uncertainties associde with turbulence refer to uncertainties originating from turbulent fluctuations at locations of el

ferest when the averaging window is not long enough.

Unlike RANS-based simulations, LES predicts time series of horizontal wind speeds and wind directions. Uncertainty associated with turbulence is estimated using time series predicted at sensor locations (sensor S1 to S7). Figure 5 presents maximal fluctuations of horizontal wind speeds and wind directions around the mean values of LES, averaged over all sensor locations, with respect to the 
averaging window. Fluctuations decrease rapidly when the averaging window increases from 1 minute to 15 minutes. Smaller reductions of these fluctuations are observed if the averaging window is further increased. Therefore, the averaging window is set to $15 \mathrm{~min}$. For this averaging window, constant wind conditions are assumed at the inlet of the RANS-based simulations used in the modelbased data-interpretation framework.

a)

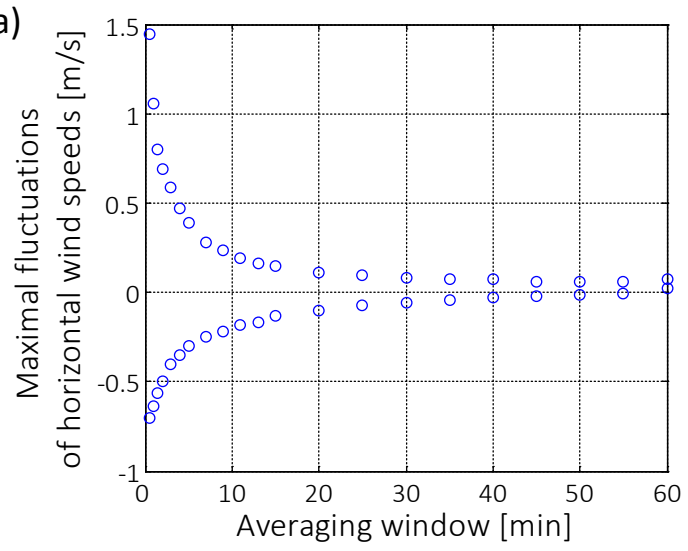

$\therefore 5$

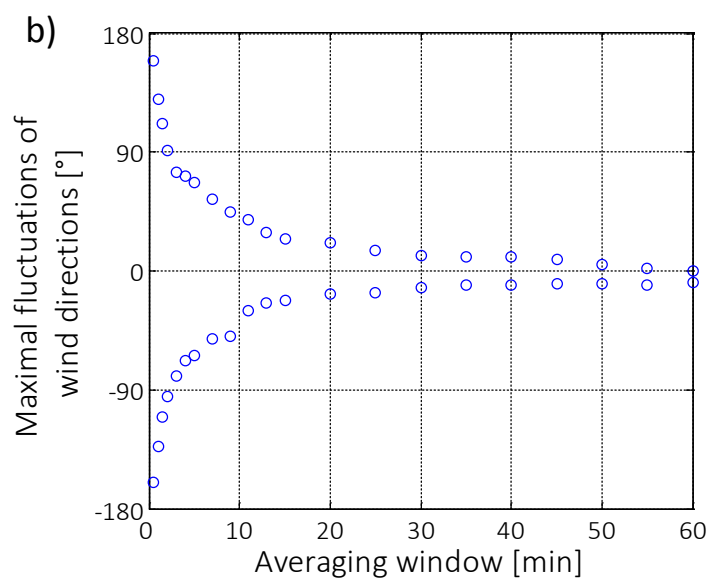

Figure 5: Maximal fluctuations of a) horizontal wind value of LES, averaged over all sensor locations, respect to the averaging window

5.3) Uncertainties associated thermal processes

Uncertainties associated with thenal processes are estimated with measurement data because modelling thermal processex CFD simulations is complex (Section 1). It would require the definition of new paraner values such as the thermal properties of surfaces and the execution of a transient simulatipor a period of several days [3].

A referend station should be placed on the roof of a building, close to the area of interest in order to $\times 2$ mead the ambient wind conditions [61]. In the urban canopy layer, the wind can be significantly affected because of the combination of low wind speeds below roof level and high differential heating between surfaces. In this work, the reference station is located on a small ridge at the rooftop of the Engineering Faculty of the National University of Singapore. Measurement data at this station are provided online (https://inetapps.nus.edu.sg/fas/geog/stationInfo.aspx). Information from the 
reference station is not used as inlet conditions in the CFD simulations because it might not be representative of the overall conditions at the inlet [30].

The steps followed to estimate the effect of thermal processes on the horizontal wind speed and the wind direction at sensors located around the CREATE Tower are described below.

1) Calculate the mean value of horizontal wind speeds and wind directions at the refereecto station.

2) Select measurement data that are taken when wind conditions at the referergestation are similar to the mean wind conditions (mean value of horizontal wind speedgand mean value of wind directions).

3) Regression analyses are employed in order to separate out effect of thermal processes from a data sample taken at different times of the day which the wind conditions above roof level are similar.

Determining the sample size is difficult becorse of two competing objectives. The sample size should be high enough in order tobble to separate out the effect of thermal processes from other sources of variability turbulence, etc.). However, the sample size should be small enough in order to hafe same ambient wind conditions measured above roof level. Thus, different sample yizes are used in the methodology. The regression analyses are expressed in Eqinoth (10) and Equation (11).

$U_{i, s}=a_{i}+b_{i, s} \times U_{r e f, s}+c_{i, s}$

Where $U_{i, s}, \vartheta_{i, s}$ and $T_{i, s}$ are vectors of horizontal wind speeds, wind directions and temperatures respectively measured at the sensor location $i$ whose sizes correspond to the number of sample s. $U_{r e f, s}$ and $\vartheta_{r e f, s}$ are vectors of horizontal wind speeds and wind directions respectively measured at the reference station whose sizes correspond to the number of sample s. $a_{i, s}, b_{i, s}, c_{i, s}, d_{i, s}, e_{i, s}$ and $f_{i, s}$ are the regression coefficients. 
4) Find out if the values of the regression coefficients, $a_{i, s}$ and $d_{i, s}$, are significantly different than 0 by performing a $t$-test [38]. If not, values of the regression coefficients are reported to be 0 .

Figure 6 presents values of the regression coefficient, $a_{i, s}$, with respect to the number of samples at sensors S5 to S8 (where temperature sensors are installed). $a_{i, s}$ is the regression coefficient determines the effect of thermal processes on the horizontal wind speed at sensor locatign asing a number of samples $s$. Values of the regression coefficient, $a_{i, s}$, are almost constapth respect to the number of samples used in the regression.

Uncertainties in the prediction of horizontal wind speed at sensor locagin are represented by uniform distributions with bounds $\left[\Delta U_{\text {low,th,i }}, \Delta U_{\text {high,th,i }}\right]$ complising Equation (12).

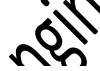

$\left[\Delta U_{\text {low }, \text { th }, i}, \Delta U_{\text {high }, \text { th }, i}\right]=\left[\min _{s}\left(a_{i, s}\right) \times\left(T_{i}-T_{\min , i}\right), \max _{s}\left(a_{i, s}\right) \times\left(T_{i}-T_{\min , i}\right)\right]$

Where $T_{i}$ is the temperature measured at sentocation $i . T_{\text {min, } i}$ is the minimal temperature measured at sensor location $i$. It is assumed thy ofermal processes have no-significant effects when the temperature measured is mininga

Wind behavior is affectulferently by thermal processes from one sensor to another as presented in Figure 6. Spatiallystributed uncertainties are thus considered in the model-based data-interpretation

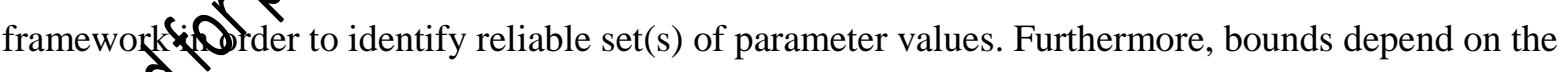
templre measured at sensor locations. Therefore, time-dependent uncertainties are also considered cर model-based data-interpretation framework. 

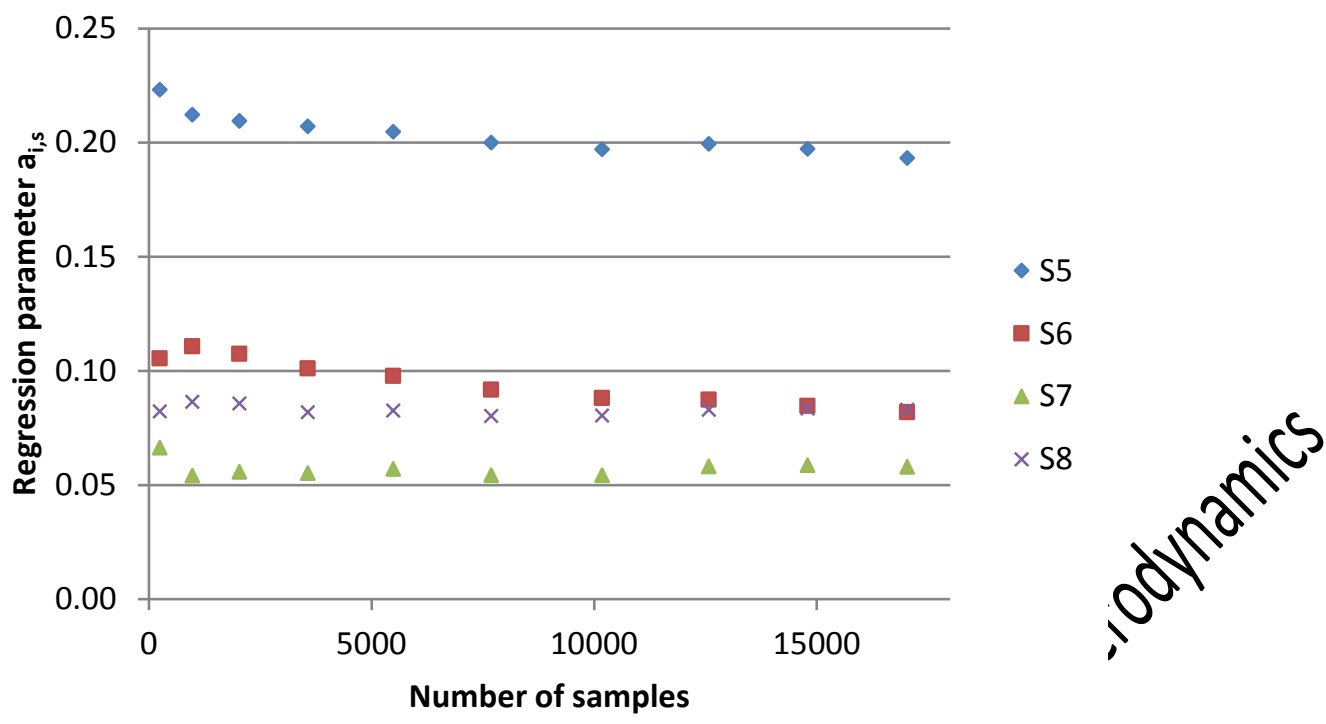

Figure 6: Regression coefficient, $a_{i, s}$, with respect to the number of samplensors S5 to S8

(averaging window $=15$ minutes)

The maximal and minimal values of the regression coefficions ${ }^{\prime} a_{i, s}$ at sensor locations are used to estimate the maximal and minimal effect of thermal pesses on the horizontal wind speed at unmeasured locations. Therefore, uncertainties at unmeasured locations are represented by uniform distributions with bounds $\left[\Delta U_{\text {low,th }}, \Delta U_{\text {low }, \text { h }}, \Delta U_{\text {high,th }}\right]=\left[\min _{i}\left(\min _{s}\left(a_{i, s}\right)\right) \times\left(\bar{T}_{i}-\bar{T}_{\text {min,i }}\right), \max _{i}\left(\max _{s}\left(a_{i, s}\right)\right) \times\left(\bar{T}_{i}-\bar{T}_{\text {min,i }}\right)\right]$

Where $\bar{T}_{i}$ is the value of $\bar{x}$

Sorme procedure has been followed in order to estimate the effect of thermal processes on the wind direction. Table 2 summarizes the minimal and maximal values of the regression coefficients at sensor locations and at unmeasured locations. The maximal and minimal values of the regression coefficients at unmeasured locations are relatively low. A stronger effect of thermal processes would be expected if measurements would be carried out in street canyons [9]. In such situations, thermal processes might need to be incorporated into the model class. 
Table 2: Minimal and maximal values of the regression coefficients at sensor locations and at unmeasured locations used to estimate uncertainty associated with thermal processes.

\begin{tabular}{|c|c|c|c|c|c|}
\hline \multirow{2}{*}{\multicolumn{2}{|c|}{$\begin{array}{c}\text { Wind } \\
\text { variable }\end{array}$}} & \multicolumn{2}{|c|}{$U[\mathrm{~m} / \mathrm{s}]$} & \multicolumn{2}{|c|}{$\vartheta\left[^{\circ}\right]$} \\
\hline & & \multirow{2}{*}{$\begin{array}{c}\min _{s}\left(a_{\text {sensor }, i, s}\right) \\
0.19\end{array}$} & \multirow{2}{*}{$\begin{array}{c}\max _{s}\left(a_{\text {sensor }, i, s}\right) \\
0.22\end{array}$} & \multirow{2}{*}{$\frac{\min _{s}\left(d_{\text {sensor }, i, s}\right)}{-1.65}$} & \multirow{2}{*}{$\begin{array}{c}\max _{s}\left(d_{\text {sensor }, i, s}\right) \\
-1.27\end{array}$} \\
\hline$\tilde{0}$ & S5 & & & & \\
\hline 苟 & S6 & 0.08 & 0.11 & 3.47 & $4 \dot{9}$ \\
\hline סे & S7 & 0.05 & 0.07 & 2.09 & 38 \\
\hline$\overline{\tilde{D}}$ & S8 & 0.08 & 0.09 & 1.21 & 1.34 \\
\hline \multirow{2}{*}{\multicolumn{2}{|c|}{ 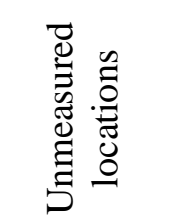 }} & $\min _{i}\left(\min _{s}\left(a_{i, s}\right)\right)$ & $\max _{i}\left(\max _{s}\left(a_{i, s}\right)\right)$ & $\min _{i}(\mathrm{~m}$ & $\max _{i}\left(\max _{s}\left(d_{i, s}\right)\right)$ \\
\hline & & 0.05 & 0.22 & & 4.60 \\
\hline
\end{tabular}

5.4) Uncertainty combination

Measurement uncertainty, uncertainty associated Dith RANS-based simulation (Section 5.1), uncertainty associated with turbulence (Section 5.4 ) and uncertainty associated with thermal processes (Section 5.3) have been combined Monte Carlo technique. Measurement uncertainty corresponds to the resolutions of the Psors mentioned in Section 4. The combination of uncertainties is illustrated in Figure 7.

Threshold bounds use falsify incorrect sets of model instances are defined using the combined uncertainty and Oonfidence level of $\varphi=95 \%$. The Šidák [39] correction is employed to adjust the

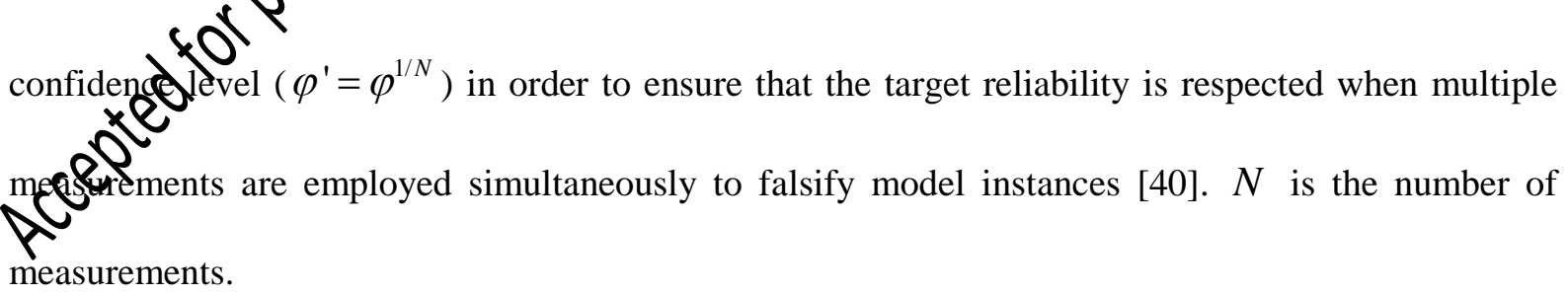




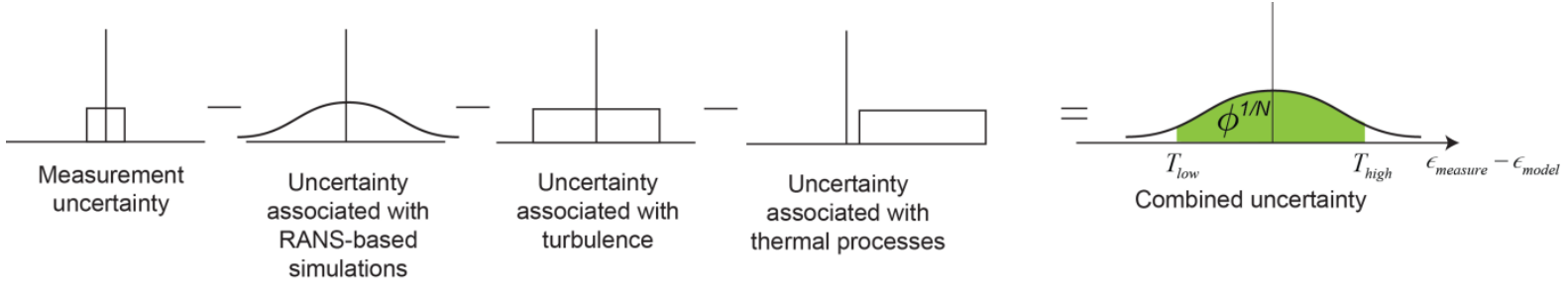

Figure 7: Uncertainty combination for the definition of threshold bounds

6) Results

6.1) Identification of parameter values of the CFD simulation

Falsification of model instances is performed every 15 minutes. Model instances are ofidate models if the differences between the measured and the predicted values of horizontal speeds and wind directions fall within threshold bounds at each and every sensor locatidection 2.2). Figure 8 presents the falsification of model instances using the horizontal wireed measured at sensor S1 at 12pm on March 11. 2014. The purple dashed line is the me value; blue lines are threshold bounds; red crosses are falsified models and green points 2010 candidate models. Falsified models that appear inside threshold bounds in Figure 8 have bealsified using measurement data at other sensor locations or using the measured wind direction this sensor location.

Threshold bounds are not the samefor with the same inlet wind speed they don't predict the same amplification factor of wind speeds at sensor S1. Furthermore,s bestic bias in modeling uncertainties has led to threshold bounds that are not centered on the rasured value.

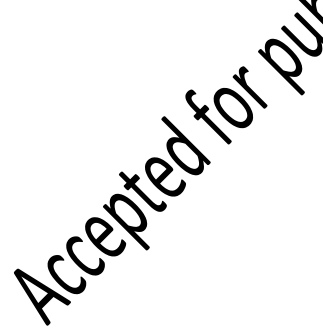



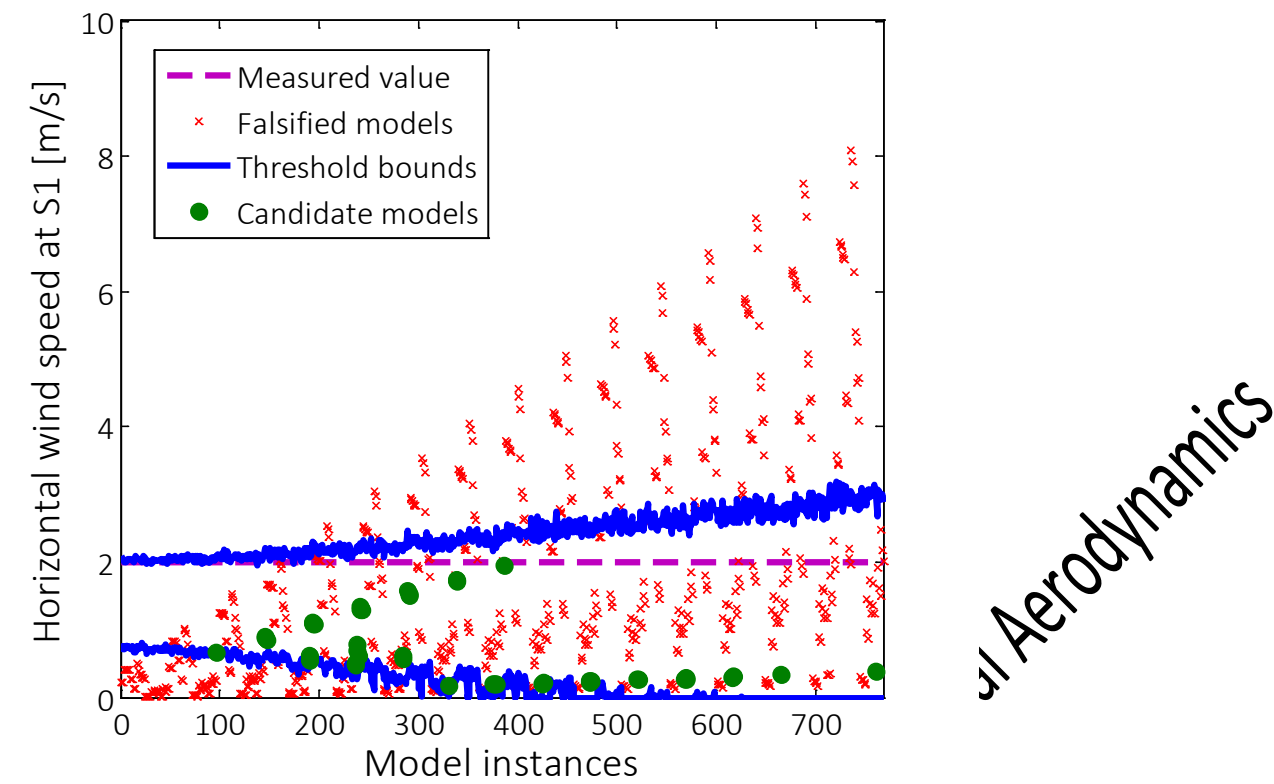

Figure 8: Falsification using the horizontal wind speed measured at senter S1 at 12pm on March 11.

2014

6.2) Predictions at unmeasured locations

A distribution of predictions can be obtained unmeasured location using the final candidate model set (Section 2.3). The likelihood we 1 or each candidate model is assumed to be equal. This distribution, which corresponds to 10 uncertainty associated with parameter values propagated through the model, is combinf with all sources of modeling uncertainty using Monte Carlo in

technique. The combinątigfor uncertainties is illustrated in Figure 9. Prediction bounds $\left[P_{\text {low }}, P_{\text {high }}\right]$ are defined using 15 inedictive distribution and a confidence level of $\varphi=95 \%$.

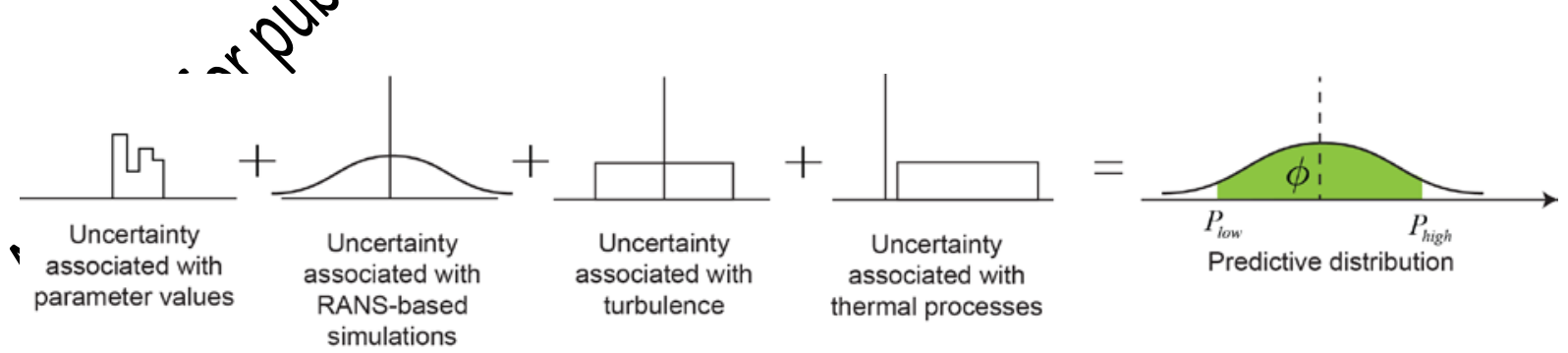

Figure 9: Uncertainty combination for predictions at unmeasured locations

Figure 10 presents predicted and measured values of horizontal wind speeds at the test sensor S8. Red points are horizontal wind speeds measured at the test sensor (sensor S8). Blue lines are prediction 
ranges of horizontal wind speeds before measurements. These ranges are obtained using the methodology presented in Figure 9; in which uncertainty associated with parameter values corresponds to the distribution of predictions obtained with the initial set of model instances. Prediction ranges vary with respect to time because of time-dependent uncertainties (uncertainties associated with thermal processes change according to the measured temperatures).

Grey lines are prediction ranges of horizontal wind speeds after measurements. Measurementa have been employed to reduce the uncertainty associated with parameter values. On aved ranges of horizontal wind-speed predictions have been decreased by 65\% when measuremptata are used. Moreover, horizontal wind speeds measured at the test sensor fall within ranges predictions 99\% of the time after measurements.

The purple dashed line is the mean values of predictions. The the measured values of horizontal wind speeds and the megroulues of predictions at the test sensor is $0.39 \mathrm{~m} / \mathrm{s}$.

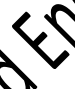

Time variability (at the scale of 15 minutes test sensor S8. Time variability is also preed in predictions. This demonstrates that the proposed framework is able to identify tependent inlet conditions. At 2:15pm, all model instances have been falsified. This might grithate from the underestimation of uncertainties associated with thermal processes, which effegro important at this time of the day. 

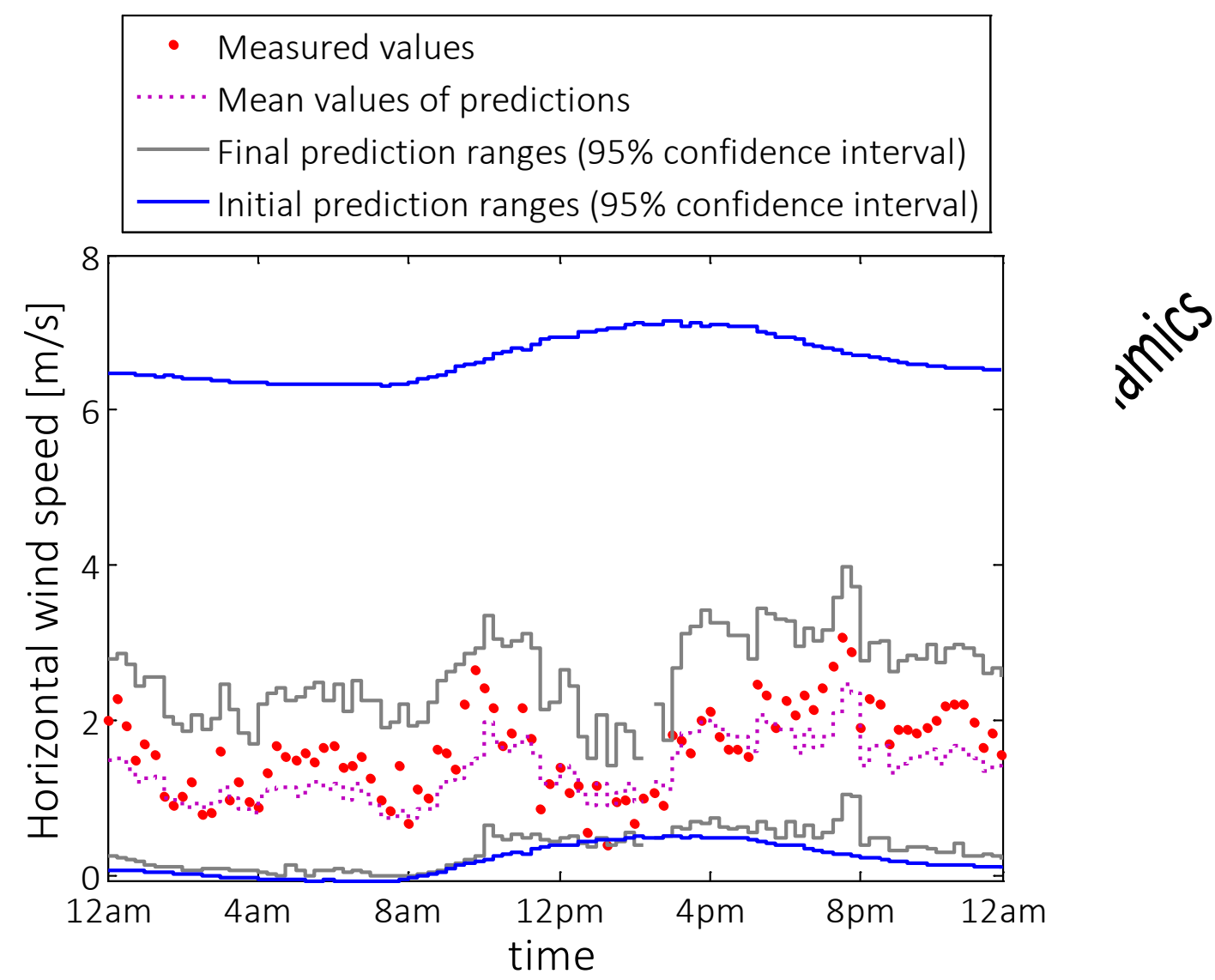

Figure 10: Predicted and measured valdof horizontal wind speeds at the test sensor S8 every 15 minutes on March 11. 2014

\section{7) Discussion}

This paper propost model-based data-interpretation framework in order to identify time-dependent sets of paraes values and predict time-dependent ranges of wind variables at unmeasured locatia Time-dependent and spatially-distributed modeling uncertainties, which affect the

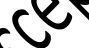

ormation content of measurement data, have been considered.

Limitations are as follows: In the present study, uncertainties associated with RANS-based simulation have been estimated with LES. Although LES has been found to be in better agreement than RANSbased simulation when compared to wind-tunnel experiments, LES is not perfectly accurate. 
Uncertainties associated with turbulence have also been estimated with LES. One simulation using LES has been executed under isothermal conditions. Otherwise definition of many new parameters such as thermal properties of surfaces would need to be defined. Furthermore, a transient simulation for a period of several days is needed to simulate such processes, which would take much longer computation time [3]. Fluctuations predicted with LES would have been larger if thermal processes were considered because of the additional turbulence generated. Moreover, a small roughness leng is employed to define the wind profiles at the inlet of LES in order to avoid unintended streanise gradients in the upstream part of the computational domain. This leads to small turbyleedeat the inlet. Thus, the turbulent fluctuations predicted by LES at sensor locations are mainly the surrounding buildings that are explicitly modelled.

Furthermore, the wind profiles used at the inlet of the CFD simulatiossumed an equilibrium boundary layer and neutral conditions. These assumptions mat be valid in all urban environments. Additional sources of uncertainties associated with the simplifications should be added in order to identify reliable sets of parameter values and predet reliable ranges of predictions at unmeasured locations. Even though uncertainties assond with these simplifications were not considered in this study, reliable ranges of predictions the still obtained at unmeasured locations (Section 6.2).

8) Conclusions

In this paper, a franifork is proposed to integrate information obtained from measurements with simulation reself The information provided by measurements is used to estimate the parameter

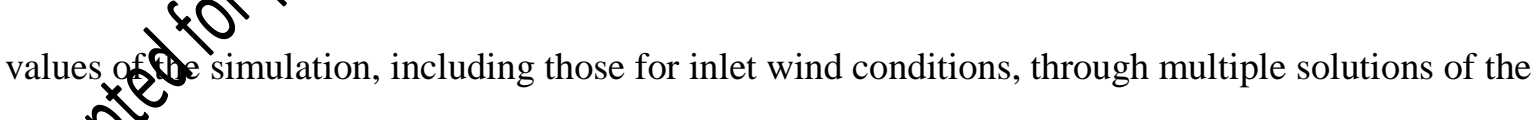
invefe problem. The information content of measurement data depends on levels of measurement and modelling uncertainties at sensor locations. Specific conclusions are:

- Differences between predictions of the RANS-based simulation and LES have been found to be large in regions of low amplification factors of wind speeds. This has led to the definition of modeling uncertainties that vary with respect to space. 
- Thermal processes significantly influence horizontal wind speeds at sensors located around the CREATE Tower. This has led to a systematic bias in the modeling uncertainty of horizontal wind speed which depends on temperature measurements.

- Reliable prediction ranges of horizontal wind speeds at unmeasured locations are obtained dynamically with the proposed framework.

- Tighter prediction ranges of horizontal wind speeds are possible using the frameisuk without compromising reliability.

Acknowledgements

This research has been conducted at the Singapore-ETH Centre foulobal Environmental Sustainability (SEC), co-funded by the Singapore National ReseardfFoundation (NRF) and ETH Zurich. The authors would like to gratefully acknowledge the surt of Prof. C. Sekhar and Prof. M. Santamouris; Prof. N.H. Wong and Asst. Prof. C. Wins for providing additional measurement equipment; Prof. em. Armin Gruen and his FCL teangor sharing their 3D models.

References

1. Defraeye, T., B. Blocken, and Curmeliet, Convective heat transfer coefficients for exterior building surfaces: Existi Rorrelations and CFD modelling. Energy Conversion and Management, 2011.52 ㄱ. p. 512-522.

2. Ghiaus, C. and F. Altrd, Natural ventilation in the urban environment: assessment and design 2005: Earocan.

3. Van Hooff, ${ }^{\top}$. B. Blocken, Coupled urban wind flow and indoor natural ventilation modelling high-resolution grid: A case study for the Amsterdam ArenA stadium. Environ (S) tal Modelling \& Software, 2010. 25(1): p. 51-65.

4. Rasolni, R. and B. Blocken, CFD simulation of cross-ventilation flow for different isolated bDiding configurations: validation with wind tunnel measurements and analysis of physical I numerical diffusion effects. Journal of Wind Engineering and Industrial Aerodynamics, 2012. 104: p. 408-418.

Franke, J., et al. Recommendations on the use of CFD in wind engineering. in Cost Action C. 2004.

6. Blocken, B. and C. Gualtieri, Ten iterative steps for model development and evaluation applied to Computational Fluid Dynamics for Environmental Fluid Mechanics. Environmental Modelling \& Software, 2012. 33: p. 1-22.

7. Tominaga, Y., et al., Comparison of various revised $k-\varepsilon$ models and LES applied to flow around a high-rise building model with 1: 1: 2 shape placed within the surface boundary layer. Journal of Wind Engineering and Industrial Aerodynamics, 2008. 96(4): p. 389-411. 
8. Yoshie, R., et al., Cooperative project for CFD prediction of pedestrian wind environment in the Architectural Institute of Japan. Journal of Wind Engineering and Industrial Aerodynamics, 2007. 95(9): p. 1551-1578.

9. Niachou, K., I. Livada, and M. Santamouris, Experimental study of temperature and airflow distribution inside an urban street canyon during hot summer weather conditions. Part II: airflow analysis. Building and Environment, 2008. 43(8): p. 1393-1403.

10. Assimakopoulos, V., C. Georgakis, and M. Santamouris, Experimental validation of a computational fluid dynamics code to predict the wind speed in street canyons for passive cooling purposes. Solar Energy, 2006. 80(4): p. 423-434.

11. Xie, X., et al., The impact of solar radiation and street layout on pollutant dispersion in straét canyon. Building and Environment, 2005. 40(2): p. 201-212.

12. Allegrini, J., V. Dorer, and J. Carmeliet, Wind tunnel measurements of buoyant flows street canyons. Building and Environment, 2013. 59: p. 315-326.

13. Beven, K., A manifesto for the equifinality thesis. Journal of hydrology, 2006. 3x( (1): p. 1836.

14. Beven, K., Environmental modelling: An uncertain future? 2008: Taylor \& Ancis.

15. Box, G.E. and G.C. Tiao, Bayesian inference in statistical analysis. Vol 2011: John Wiley \& Sons.

16. Goulet, J.-A., S. Coutu, and I.F.C. Smith, Model falsification digghlis and sensor placement for leak detection in pressurized pipe networks. Advanced Egoforing Informatics, 2013. 27(2): p. 261-269.

17. Goulet, J.-A. and I.F. Smith, Structural identification witerystematic errors and unknown uncertainty dependencies. Computers \& Structures 13. 128: p. 251-258.

18. Goulet, J.-A., C. Michel, and I.F.C. Smith, Hybri error-domain structural identification using ambient vibration monito 2 . Mechanical Systems and Signal Processing, 2012. 37(1): p. 199-212.

19. Goulet, J.-A. and I.F. Smith. Extended if orm distribution accounting for uncertainty of uncertainty. in International Conferde on Vulnerability and Risk Analysis and Management/Fifth Internationalosium on Uncertainty Modeling and Analysis, Maryland, USA. 2011.

20. Cox, M.G. and B.R. Siebert he use of a Monte Carlo method for evaluating uncertainty and expanded uncertainty.

21. Fluent, A., 14.5. Thea Guide, 2012. 117.

22. Gruen, A., et al., Processing of Uav Imagery and Terrestrial Mobile Mapping System Data for Very Resolution City Modeling. ISPRS-International Archives of the Photogramint Remote Sensing and Spatial Information Sciences, 2013. 1(2): p. 175-182.

23. Franke, 1 al., The COST 732 Best Practice Guideline for CFD simulation of flows in the urbansenironment: a summary. International Journal of Environment and Pollution, 2011. 44 (1.0. 419-427.

24. $x$, T.-H., et al., A new $k-\varepsilon$ eddy viscosity model for high reynolds number turbulent flows.

Computers \& Fluids, 1995. 24(3): p. 227-238.

Franke, J., et al., Best practice guideline for the CFD simulation of flows in the urban environment 2007. Hamburg: COST Action. 732.

26. Richards, P. and R. Hoxey, Appropriate boundary conditions for computational wind engineering models using the $k-\epsilon$ turbulence model. Journal of Wind Engineering and Industrial Aerodynamics, 1993. 46: p. 145-153.

27. Launder, B.E. and D. Spalding, The numerical computation of turbulent flows. Computer methods in applied mechanics and engineering, 1974. 3(2): p. 269-289.

28. Blocken, B., T. Stathopoulos, and J. Carmeliet, CFD simulation of the atmospheric boundary layer: wall function problems. Atmospheric environment, 2007. 41(2): p. 238-252. 
29. Wieringa, J., Updating the Davenport roughness classification. Journal of Wind Engineering and Industrial Aerodynamics, 1992. 41(1): p. 357-368.

30. Schatzmann, M. and B. Leitl, Issues with validation of urban flow and dispersion CFD models. Journal of Wind Engineering and Industrial Aerodynamics, 2011. 99(4): p. 169-186.

31. Blocken, B. and J. Persoon, Pedestrian wind comfort around a large football stadium in an urban environment: CFD simulation, validation and application of the new Dutch wind nuisance standard. Journal of Wind Engineering and Industrial Aerodynamics, 2009. 97(5): p. 255-270.

32. Papadopoulou, M., et al., Optimal sensor placement for time-dependent systems: application to wind studies around buildings. Journal of Computing in Civil Engineering, (under reviewt.

33. Martilli, A., J.L. Santiago, and F. Martín, Micrometeorological modelling in urban areas. de la Tierra, 2008. 19: p. 133-145.

34. Mathey, F., et al., Assessment of the vortex method for large eddy simulation inled conditions. Progress in Computational Fluid Dynamics, An International Journa 206.6 (1): p. 58-67.

35. Gousseau, P., et al., CFD simulation of near-field pollutant dispersion on grid: a case study by LES and RANS for a building group in downtown treal. Atmospheric environment, 2011. 45(2): p. 428-438.

36. Germano, M., et al., A dynamic subgrid-scale eddy viscosity med Physics of Fluids A: Fluid Dynamics, 1991. 3: p. 1760.

37. Vernay, D.G., B. Raphael, and I.F. Smith, Augmenting simans of airflow around buildings using field measurements. Advanced Engineering InfogRatics, 2014.

38. Zar, J.H., Biostatistical analysis. 2nd. Prentice Hall b. 1984.

39. Šidák, Z., Rectangular confidence regions for the of multivariate normal distributions. Journal of the American Statistical Associatio 1967. 62(318): p. 626-633.

40. Goulet, J.A. and I.F. Smith, Performance measurement system design for structural identification. Journal of Computing infit Engineering, 2012. 27(4): p. 427-436.

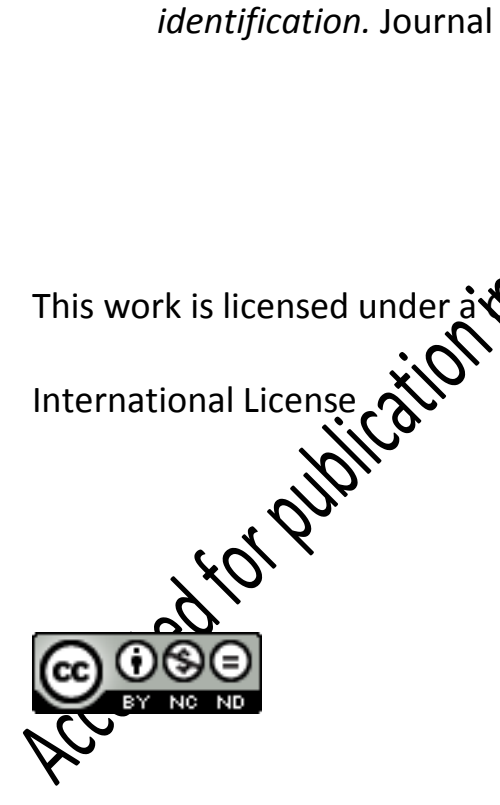

